\title{
ASO Author Reflections: Pelvic Exenteration for Patients with Primary and Recurrent Pelvic Cancer
}

\author{
Yakup Kulu, MD \\ University of Heidelberg, Heidelberg, Germany
}

\section{PAST}

Pelvic exenteration to remove primary locally advanced or recurrent pelvic tumors is a complex surgical procedure with potential high morbidity and mortality. Margin negativity is an accepted prognostic factor. While margin negativity is important, data on the role of disease stage is conflicting. A recent study demonstrated that survival of patients with primary and recurrent disease was similar 3 years after pelvic exenteration, ${ }^{1}$ while two other studies, both with limited patient numbers, showed survival was worse for patients with recurrent disease, regardless of resection status. ${ }^{2,3}$ Patients with extrapelvic disease are rarely included in studies on pelvic exenteration; however, we also included patients with extrapelvic disease in our study, which aimed to assess the impact of disease stage on long-term oncological outcomes after pelvic exenteration in a large, single-center cohort.

\section{PRESENT}

The present study shows that survival is different between patients with primary and recurrent disease 3 years after pelvic exenteration. ${ }^{4}$ The oncological outcome of patients with recurrent disease is worse than that of patients with primary disease. This remains true if only curatively resected patients are considered. Local and

ASO Author Reflections is a brief invited commentary on the article "Promising Long-Term Outcomes After Pelvic Exenteration", Ann Surg Oncol. 2018.

\section{(C) Society of Surgical Oncology 2019}

First Received: 25 February 2019;

Published Online: 6 March 2019

Y. Kulu, MD

e-mail: yakup.kulu@med.uni-heidelberg.de systemic recurrence rates after pelvic exenteration were only analyzed in curatively resected patients. Disease stage was the only significant prognostic factor. Not surprisingly, extrapelvic disease was an independent prognostic factor of poor prognosis; however, a well-selected subset of patients with extrapelvic disease benefits from pelvic exenteration. Survival is poor without surgery in patients with pelvic malignancies, therefore surgery is justified for patients who seem curatively resectable.

\section{FUTURE}

Disease stage plays an important role in oncological outcome following pelvic exenteration, and recurrent disease is associated with a worse outcome. While adjuvant treatment and follow-up examinations after surgical resection are well-defined for primary disease, additional therapy regimens and intensified follow-up examinations for early detection in recurrent patients are needed. To address this, prospective multicenter studies should be performed.

DISCLOSURE Yakup Kulu has no conflicts of interest to disclose.

\section{REFERENCES}

1. Bhangu A, Ali SM, Brown G, et al. Indications and outcome of pelvic exenteration for locally advanced primary and recurrent rectal cancer. Ann Surg 2014; 259: 315-322.

2. Nielsen MB, Rasmussen PC, Lindegaard JC, et al. A 10-year experience of total pelvic exenteration for primary advanced and locally recurrent rectal cancer based on a prospective database. Colorectal Dis 2012; 14: 1076-1083.

3. Rottoli M, Vallicelli C, Boschi L et al. Outcomes of pelvic exenteration for recurrent and primary locally advanced rectal cancer. Int J Surg 2017; 48: 69-73.

4. Kulu Y, Mehrabi A, Khajeh E, et al. Promising long-term outcomes after pelvic exenteration. Ann Surg Oncol. Epub 5 Dec 2018. https://doi.org/10.1245/s10434-018-07090-0.

Publisher's Note Springer Nature remains neutral with regard to jurisdictional claims in published maps and institutional affiliations. 